\title{
Microbiological monitoring in patients with advanced ovarian cancer before and after cytoreductive surgery - a preliminary report
}

\author{
Maria Szymankiewicz ${ }^{1}$, Krzysztof Koper ${ }^{2,5}$, Konrad DziobeK ${ }^{3}$, \\ ZBIGNIEW KoJS ${ }^{3}$, LUKASZ WiCHEREK ${ }^{4,5 *}$
}

\footnotetext{
${ }^{1}$ Department of Microbiology, Professor Franciszek Lukaszczyk Oncology Center, Bydgoszcz, Romanowskiej 2, 85-796 Bydgoszcz, Poland

${ }^{2}$ Department of Oncology, Professor Franciszek Lukaszczyk Oncology Center, Bydgoszcz, Romanowskiej 2, 85-796 Bydgoszcz, Poland

${ }^{3}$ Department of Gynecologic Oncology, Maria Sklodowska-Curie Memorial Cancer Centre and Institute of Oncology in Warsaw, Division in Krakow, Garncarska 11, 31-115 Krakow, Poland

${ }^{4}$ Clinical Department of Gynecologic Oncology, Professor Franciszek Lukaszczyk Oncology Center, Bydgoszcz, Romanowskiej 2, 85-796 Bydgoszcz, Poland

${ }^{5}$ Department of Oncology, Radiotherapy and Oncological Gynecology, Ludwik Rydygier Collegium Medicum, Nicolaus Copernicus University, Bydgoszcz, Romanowskiej 2, 85-796 Bydgoszcz, Poland
}

\section{ARTICLE INFO \\ Received 26 September 2017 Accepted 29 December 2017}

\section{Keywords:}

microbiological monitoring, multidrug-resistant organisms, advanced ovarian cancer, cytoreductive surgery.

\begin{abstract}
Multidrug-resistant organisms (MDROs) are becoming an increasing problem in hospitals. It is believed that screening patients for the incidence of MDROs prior to hospital admission not only allows for the proper management of infection following medical procedures, but can also potentially reduce the transmission of these bacteria to other patients.

The aim of this study was to assess the carriers of selected MDROs in the gastrointestinal tract among patients with advanced ovarian cancer admitted to the hospital for cytoreductive surgery and to estimate the possible relationship between rectal colonization with these organisms and nosocomial infections.

From December 2013 to May 2014, we evaluated the colonization with VRE (vancomycinresistant Enterococcus), E. coli KPC+ (class A carbapenemase producing Escherichia coli), E. coli $\mathrm{MBL}+$ (class B carbapenemase, metallo- $B$ lactamase producing Escherichia coli), and E. coli ESBL+ (extended-spectrum B-lactamase producing Escherichia coli) in 42 patients. The patients were divided into two subgroups corresponding to the extent of their surgery: the first subgroup consisted of patients with large bowel resection $(\mathrm{n}=18)$ and the second subgroup of patients without resection $(n=24)$. A rectal swab was taken within 24 hours of admission. Perioperative infectious complications were analyzed for the first 90 days following surgery with regard to the type of infection and the occurrence of examined MDROs.

In our study, $2.4 \%$ of all patients (23.8/1,000 hospitalizations) were colonized with ESBL - producing Escherichia coli: $0.0 \%$ in the first subgroup and $4.2 \%$ in the second subgroup, respectively. We did not identify any patients who were colonized with VRE, E. coli MBL+, or E. coli KPC+. Surgical site infections were seen in 8 (19.1\%) out of 42 patients. We were, therefore, unable to confirm a relationship between MDROs colonizing the large bowel and the etiological agents of perioperative infections. However, despite the lack of identification of MDROs as etiological agents of postoperative infection, the risk of serious infectious complications, combined with the changing epidemiological situation, means that microbiological monitoring should be performed in patients with ovarian cancer before and after cytoreductive surgery.
\end{abstract}

\footnotetext{
* Corresponding author

e-mail: agkola@interia.pl
} 


\section{INTRODUCTION}

Cytoreductive surgery in patients with advanced ovarian cancer is often complicated by infection. Extensive, longterm surgical procedures with long periods of tissue exposure, including large bowel resection, are risk factors for infection. Additionally, intensive care unit treatment with invasive procedures is conducive to the emergence of infectious complications [1-3]. The most common etiological agents of infection are Gram-negative rods and enterococci, often originating from the patient's own flora [3-5]. The reservoir of bacteria responsible for endogenous infections is the gastrointestinal tract, as the colon is most commonly colonized with microorganisms. The intestinal microbiota depends on a number of factors, but the most important is antibiotic therapy. The use of broad-spectrum antibiotics, in particular, can change the intestinal microbiome structure and increase the rate of bacteria resistant to antibiotics [6-7]. Furthermore, previous stays in a hospital environment where resistant microorganisms occur favor change in the microbiota [8].

According to the recommendations of the Centers for Disease Control and Prevention, microbiological screening for multidrug-resistant microorganisms (MDROs) in oncological patients who are considered at high-risk of developing an infection should be performed on admission to the hospital [9]. Due to undergoing bowel resection, patients have an increased risk of developing infections from microorganisms occurring in the digestive tract following cytoreduction. Risk factors for colonization with MDROs in these patients include previous hospitalizations, antibiotic treatments and co-morbidities [3-5].

Available publications rarely, however, discuss multidrug-resistant Gram-negative rods and screening cultures in patients before oncology surgery, but some authors have pointed out that preoperative screening can reduce empirical treatment with a targeted therapy [10]. Microbiological screening also allows the patient-carriers of MDRO to be identified and action that might limit the transmission of these microorganisms to other susceptible patients to be taken. However, the experience of some authors shows that the impact of screening is evident only in the case of the epidemic spread of MDROs [9]. According to other authors, most patients colonized with MDROs are not diagnosed through clinical cultures, so they can constitute a hidden reservoir of MDROs. A much higher rate of MDROs is reported for targeted screening in high-risk patients [11-13].

The aim of our study was to evaluate the prevalence of the positive results of vancomycin- resistant enterococci and carbapenemase or extended-spectrum B-lactamase producing Escherichia coli among patients with advanced ovarian cancer admitted to the hospital for cytoreductive surgery, and to assess the possible relationship between colonization with MDROs detected in screening and the etiological agents of postoperative infections.

\section{MATERIAL AND METHODS}

The prospective study included 42 patients with advanced ovarian cancer admitted to the Clinical Department of
Gynecologic Oncology at the Oncology Center in Bydgoszcz during the 6-month period between December 2013 and May 2014. These patients underwent primary $(\mathrm{n}=17)$ or secondary $(n=25)$ cytoreductive surgery. From this group, two subgroups were selected, differing, among others factors, in the extent of the surgery performed. In the first subgroup of 18 patients, the surgery included large bowel resection; in the second subgroup of 24 patients, there was no interruption of gastrointestinal continuity. The median age of the patients was 53 in the first subgroup and 57 in the second. On the day of admission to the hospital, rectal swabs for VRE (vancomycin-resistant Enterococcus), E. coli KPC+ (class A carbapenemase producing Escherichia coli), E. coli $\mathrm{MBL}+$ (class B carbapenemase, metallo- $\beta$ lactamase producing Escherichia coli), and E. coli ESBL+ (extended-spectrum ß-lactamases producing Escherichia coli) were taken with the patients' consent. The material was then transferred to the Department of Microbiology where the chromogenic plates were cultured. These included: Brilliance VRE Agar, Brilliance ESBL and Brilliance CRE Agar (Oxoid, UK). The plates were incubated under the aerobic conditions specified in the manufacturer's instructions. In the case of microbial growth, identification, and resistance mechanisms were determined in accordance with the applicable procedures, using the VITEK 2 Compact (bioMérieux, USA) and the disc-diffusion method. The results were interpreted according to current EUCAST breakpoints.

In the case of diagnosis of infection within 90 days of surgery, clinical material corresponding to the site of infection was collected in accordance with the criteria of the Centers for Disease Control and Prevention. A microbiological diagnostic was then conducted following standard research procedures applicable in the Department of Microbiology of the Oncology Center in Bydgoszcz, and based on both current recommendations and recognized methodical publications.

The study was approved by the Bioethics Committee of the Collegium Medicum Nicolaus Copernicus University in Bydgoszcz.

\section{RESULTS}

In the screening conducted on the day of admission to the hospital, we did not find any VRE nor did we find E. coli $\mathrm{KPC}+$ or $\mathrm{MBL}+$ in any of the patients in the two examined subgroups. Likewise, E. coli ESBL+ was not found in any patients belonging to the first subgroup. In the second subgroup, the presence of $E$. coli $\mathrm{ESBL}+$ was confirmed in 1 out of 24 patients $(4.2 \%)$. Additionally, in the first subgroup, postoperative wound infection was confirmed in 5 out of 18 patients $(27.8 \%)$, including one case with multiple infections (wound infection and organ /space infection). In the second subgroup, infection of the postoperative wound was observed in 3 out of 24 patients $(12.5 \%)$. We did not observe infections of any other type. Table 1 shows postoperative infectious complications. When it came to etiology, we found no correlation between isolated MDROs present before surgery and the etiologic agent causing the postoperative infection. None of the examined patients developed a postoperative infection that was caused by the 
tested multidrug-resistant microorganisms originating from others sources.

Table 1. Postoperative infections complications

\begin{tabular}{|c|c|c|c|c|}
\hline \multirow{2}{*}{ Variables } & \multirow{2}{*}{$\begin{array}{c}\text { Number } \\
\text { of patients } \mathrm{N}\end{array}$} & \multicolumn{3}{|c|}{ Type of infection } \\
\hline & & SSI & UTI & BSI \\
\hline $\begin{array}{c}\text { Subgroup } 1 \\
\text { (patients with large } \\
\text { bowel resection) }\end{array}$ & 18 & $5(27.8 \%)$ & 0 & 0 \\
\hline $\begin{array}{c}\text { Subgroup } 2 \\
\text { (patients without } \\
\text { large bowel resection) }\end{array}$ & 24 & $3(12.5 \%)$ & 0 & 0 \\
\hline Overall & 42 & $8(19.1 \%)$ & 0 & 0 \\
\hline
\end{tabular}

UTI - Urinary tract infections

BSI - Bloodstream infections

\section{DISCUSSION}

Infections after aggressive surgical procedures occur relatively frequently among ovarian cancer patients, the most common types being surgical site infections, urinary tract infections, and bacteremia/sepsis. Infectious complications are considered a cause of mortality in this group of patients $[1,5,10,14-17]$. If the infections are caused by MDROs, the options for antibiotics are limited, and, as a consequence, the mortality rate increases compared to the mortality of patients whose infections are caused by antibiotic-susceptible microorganisms. Higher treatment costs and a longer hospital stay are also typically involved $[9,18]$. So far, little research has been done on the importance of MDRO colonization and the significance of screening for MDROs among patients undergoing extensive surgical procedures. Indeed, the available studies mainly concern hematologic patients, such as transplant recipients and dialysis patients [19]. Because evidence shows that prior colonization of the gastrointestinal tract with MDROs increases the overall risk of postoperative infection with these microorganisms [18-20], we evaluated the colonization with the most important MDROs in the patients with solid tumors who qualified for extensive surgery with a possible opening of the gastrointestinal tract.

When the risk for colonization with this organism is high, screening for VRE is recommended. According to Tacconelli et al. [21], screening on admission to the hospital should be conducted in case of positive risk factors, such as use of antibiotics within the last 30 days, age $>60$ years, hospitalization within the previous year, and transfer from another hospital. In studies by Matar, et al. [20] on the occurrence of VRE colonization and subsequent infection in about 2,000 patients with hematologic malignancies or who are bone marrow transplant recipients, the presence of VRE was confirmed in $4.7 \%$ of patients. Moreover, bacteremia VRE was several times more common in patients with hematologic malignancies than in those with solid tumors, although for the latter group, the authors did not evaluate the frequency of VRE colonization. There is evidence that mucositis of a certain severity, prior use of vancomycin, and surgery on the gastrointestinal tract are significant risk factors for the development of VRE bacteremia [22-23]. In contrast to the study by Matar et al. cited above [20], a study on a smaller group of patients by Zaas et al. [23] found no statistically significant differences in the incidence of colonization and VRE bacteremia in patients relative to tumor type (solid versus other types of tumors). As there were no cases of bacteremia/sepsis in our study, we were unable to confirm any relationship between colonization and later infections. In contrast, some authors [24-25] question the validity of screening as ineffective in limiting transmission and VRE infections. It also seems that there may be problems with following the screening recommendations as information about prior antibiotic use or VRE occurrence in other hospitals is not always available.

In our study, we were unable to confirm the occurrence of VRE strains either on hospital admission or after cytoreductive surgery. This may be related to a hospital-based antibiotic policy regarding the restrictive use of vancomycin and to the local epidemiological situation of both the hospital and the region (low-incidence density of VRE). However, it is worth emphasizing that the admission of a single VREcolonized patient can lead to the rapid spread of VRE on a ward and, as a consequence, to long-standing infections on the endemic level [26-27].

Infections caused by Gram-negative rods producing ESBL are quite common. Typical reasons for the frequent occurrence of microorganisms with this resistance mechanism are prior use of antibiotics (especially cephalosporins 3rd generation and fluoroquinolones), invasive procedures, and hospitalization in intensive care units [11,28-29]. The reservoir of Gram-negative rod ESBL+, as in the case of VRE, is the carrier's own digestive tract. Notably, carriage can be long-term. According to a study by Freeman et al. [30], 75.0\% of all carriers remained colonized at one year and the median duration of carriage is about 3 years. However, when it comes to the effectiveness of screening for ESBL+ Enterobacteriaceae, research has yielded ambiguous results. On the one hand, a study by Thouverez [31] demonstrates that in the case of a sporadic, non-epidemic occurrence of ESBL+Enterobacteriaceae, clinical cultures are sufficient to control the occurrence of the bacteria. On the other hand, based on the results of a study on the incidence of infections after cytoreductive surgery and hyperthermic intraperitoneal chemotherapy conducted by Vall et al. [10], the use of a standardized protocol in all patients enrolled for a combined procedure, including preoperative screening for ESBL and VRE is recommended.

In European countries, the rate of $E$. coli $\mathrm{ESBL}+$ carriage in the gastrointestinal tract is highly variable, ranging from $2.7 \%$ to $30.0 \%$ of all patients, and may also occur in people who have never been hospitalized [28-29,32]. In our study, we confirmed a rather low prevalence of $E$. coli ESBL+ carriers, identifying one carrier among 42 examined patients. The patient who has confirmed the presence of this microorganism has had numerous contacts with healthcare before, including surgical procedures.

The risk factors for colonizing Gram-negative rod-producing carabapenemases are the same as for the previously mentioned microorganisms, but in this case, colonization is primarily associated with hospitalization in a facility with high incidence of infections caused by carbapenemase-producing rods [33]. About $39.0 \%$ of all patients remained colonized with Gram-negative-rod-producing carabapenemases for up to one year. In cases of symptomatic infections and multiple hospitalizations, the median colonization time is 
higher and may last about 2 years [34]. In our study, we did not confirm the presence of carbapenemase-producing $E$. coli strains. Nevertheless, due to the great potential of these organisms and the changing epidemiological situation in Poland resulting from the rapid spread of carbapenemaseproducing strains, the recommendations in case of identification of these strains should be followed [35].

We found no link between the screening results and the appearance of infection after the surgery, regardless of the etiological agent. However, in the subgroup of patients with large bowel resection, we did not observe colonization with the examined MDROs on admission; therefore, we cannot confirm whether there is an increased risk for the emergence of infection from MDROs in case of opening a gastrointestinal tract colonized with MDROs. Still it is known that in case of resection of the gastrointestinal tract, the most common etiologic agents of intra-abdominal infections are bacteria derived from the gastrointestinal tract $[3,5,10]$.

The results of our preliminary study indicate that MDROs are infrequent in patients enrolled for cytoreductive surgery; however, these results relate to a single institution. Our work has a number of other limitations. First of all, it concerns a small group of patients admitted to the hospital in order to undergo cytoreductive surgery for ovarian cancer. Secondly, it involves a relatively brief period of observation. In addition, the risk factors for colonization by the MDROs were not collected, but were instead based on the assumption that primary disease, prior hospitalizations, and invasive procedures in these patients may result in increased incidence of MDROs. It appears, however, that among the examined patients, there were likely few, if any, who had ever had contact with the environment in which the carbapenemaseproducing E. coli or VRE strains were present.

Considering the relatively small group of patients, it would appear that in order to confirm that the MDROs originated from the digestive tract of operative patients and are not significant etiological agents of postoperative infections, the study should be repeated on a larger group of patients, taking into account the risk factors for MDROs.

\section{CONCLUSIONS}

1. Carriers of ESBL-producing $E$. coli were infrequent among patients with ovarian cancer admitted to the hospital for cytoreductive surgery. Carriers of carbapenemase-producing strains (KPC and MBL) and vancomycin-resistant enterococci were not identified.

2. Due to the risk of serious infectious complications caused by MDROs and a variable epidemiological situation, it would seem reasonable to conduct microbiological monitoring not only after cytoreductive surgery, but also on admission to the hospital.

\section{REFERENCES}

1. Gersetein CG, Damhuis RAM, de Vries M, Reedijk A, Burger CW, Kooi GS. Causes of postoperative mortality after surgery for ovarian cancer. Eur J Cancer. 2009;45:2799-803.
2. Chi DS, Eisenhauer EL, Zivanovic O, SonodaY, Abu-Rustum NR, Levine DA, et al. Improved progression-free and overall survival in advanced ovarian cancer as a result of a change in surgical paradigm. Gynecol Oncol. 2009;114:26-31.

3. Capone A, Valle M, Proietti F, Federici O, Garofalo A, Petrosillo N. Postoperative infections in cytoreductive surgery with hyperthermic intraperitoneal intraoperative chemotherapy for peritoneal carcinomatosis. J Surg Oncol. 2007;96:507-13.

4. Dazza M, Schwarz L, Coget J, Frebourg N, Wood G, Huet E, et al. Does intraoperative systematic bacterial sampling during complete cytoreductive surgery (CRS) with hyperthermic intraoperative peritoneal chemotherapy (HIPEC) influence postoperative treatment? A new predictive factor for postoperative abdominal infectious complications. World J Surg. 2016;40:3035-43.

5. Matsuo K, Prather CP, Ahn EH, Eno ML, Tierney KE, Yessaian AA , et al. Significance of perioperative infection in survival of patients with ovarian cancer. Int J Gynecol Cancer. 2012;22:245-53.

6. Wołkowicz T, Januszkiewicz A, Szych J. Mikrobiom przewodu pokarmowego i jego dysbiozy jako istotny czynnik wpływający na kondycję zdrowotną organizmu człowieka. Med Dośw Mikrobiol. 2014;66:223-35.

7. Jernberg C, Löfmark S, Edlund C, Jansson JK. Long-term impacts of antibiotic exposure on the human instestinal microbiota. Microbiology. 2010;156:3216-23.

8. Esposito S, Capuano A, Noviello S, Mazzeo F, Ianniello F, Filippelli A. Modification of patients' endogenous bacterial flora during hospitalization in a large teaching hospital in Naples. J Chemother. 2003;15:568-73.

9. Siegel JD, Rhinehart E, Jackson M, Chiarello L. The Healthcare Infection Control Practices Advisory Committee. Management of multidrug-resistant organisms in health care settings, 2006. Am J Infect Control. 2007;3:165-93. www.cdc.gov/infectioncontrol/ guidelines/mdro, last update: February, 15, 2017.

10. Valle M, Federici O, Carboni F, Toma L, Gallo MT, Prignano G, et al. Postoperative infections after cytoreductive surgery and HIPEC for peritoneal carcinomatosis: Proposal and results from a prospective protocol study of prevention, surveillance and treatment. EJSO. 2014;40:950-6.

11. Harris AD, Nemoy L, Johnson JA, Martin-Carnahan A, Smith $\mathrm{DL}$, Standiford $\mathrm{H}$, et al. Co-carriage rates of vancomycin-resistant Enterococcus and extended-spectrum beta lactamase-producing bacteria among a cohort of intensive care unit patients: implications for an active surveillance program. Infect Control Hosp Epdemiol. 2004;25:105-8.

12. Ostrowsky BE, Venkataraman L, D’Agata EM, Gold HS, DeGirolami PC, Samore MH. Vancomycin-resistant enterococci in intensive care units: high frequency of stool carriage during a non-outbreak period. Arch Intern Med. 1999;159:1467-72.

13. Warren DK, Nitin A, Hill C, Fraser VJ, Kollef MH. Occurrence of co-colonization or co-infection with vancomycin-resistant enterococci and methicillin-resistant Staphylococcus aureus in a medical intensive care unit. Infect Control Hosp Epidemiol. 2004;25: 99-104.

14. Tran CW, McGree ME, Weaver AL, Martin JR, Lemens MA, Cliby WA, et al. Surgical site infection after primary surgery for epithelial ovarian cancer: predictors and impact on survival. Gynecol Oncol. 2015;136:278-84.

15. Rafii A, Stoeckle E, Jean-Laurent M, Ferron G, Morice P, Houvenaeghel G, et al. Multi-center evaluation of post-operative morbidity and mortality after optimal cytroreductive surgery for advanced ovarian caner. Plose One. http://dx.doi.org/10.1371/ journal.pone.0039415, 2012 (accessed 23.07.2012).

16. Sugarbaker PH. Peritonectomy procedures. Ann. Surg. 1995;221:29-42.

17. Sugarbaker PH, Alderman R, Edwards G, Marquardt CE, Gushchin V, Esquivel J, et al. Prospective morbidity and mortality assessment of cytoreductive surgery plus perioperative intraperitoneal chemotherapy to treat peritoneal dissemination of appendiceal mucinous malignancy. Ann Surg Oncol. 2006;13:635-44. 
18. Capitano B, Nicolau DP. Evolving epidemiology and costs of resistance to antimicrobial agents in long-term care facilities. J Am Med Dir Assoc. 2003;4(3 Suppl):S90-9.

19. Alevizakos M, Gaitanidis A, Nasioudis D, Tori K, Flokas ME, Mylonakis E. Colonization with vancomycin-resistant enterococci and risk for bloodstream infection among patients with malignancy: a systemic review and meta-analysis. Open Forum Infect Dis.2017;4 (1):ofw246, Published online 2016 Dec. 7. https://doi.org/10.1093/ ofid/ofw246.

20. Matar MJ, Tarrand J, Raad I, Rolston KV. Colonization and infection with vancomycin-resistant enterococcus among patients with cancer. Am J Infect Control. 2006;34:534-6.

21. Tacconelli E. New strategies to identify patients harbouring antibiotic-resistant bacteria at hospital admission. Clin Microbiol Infect. 2006;12:102-9.

22. Kuehnert MJ, Jernigan JA, Pullen AL, Rimland D, Jarvis WR. Association between mucositis severity and vancomycin-resistant entrococcal bloodstream infection in hospitalized cancer patients. Infect Control Hosp Epidemiol. 1999;20: 660-3.

23. Zaas AK, Song X, Tucker P, Perl TM. Risk factors for development of vancomycin-resistant enterococcal bloodstream infection in patients with cancer who are colonized with vancomycin-resistant enterococci. Clin Infect Dis. 2002;35:1139-46.

24. Almyroudis NG, Osawa R, Samonis G, Wetzler, Wang ES, McCarthy PL, et al. Discontinuation of systematic surveillance and contact precautions for vancomycin-resistant Enterococcus (VRE) and its impact on the incidence of VRE faecium bacteremia in patients with hematologic malignancies. Infect Control Hosp Epidemiol. 2016;37:398-403.

25. De Angelis G, Cataldo MA, De Waure C, Venturiello S, La Torre G, Cauda R, et al. Infection control and prevention measures to reduce the spread of vancomycin-resistant enterococci in hospitalized patients: a systemic review and meta-analysis. J Antimicrob Chemother. 2014;69:1185-92.

26. Farr BM. Hospital wards spreading vancomycin-resistant enterococci to intensive care units: returning coals to Newcastle. Crit Care Med, 1998;26:1942-3.
27. Weinstein JW, Roe M, Towns M, Sanders L, Thorpe JJ, Corey GR, et al. Resistant enterococci: a prospective study of prevalence, incidence, and factors associated with colonization in a university hospital. Infect Control Hosp Epidemiol. 1996;17:36-41.

28. Kang ChI, Wi YM, Lee MY, Ko KS, Chung DR, Peck KR, et al. Epidemiology and risk factors of community onset infections caused by extended-spectrum ß-lactamase-producing Escherichia coli strains. J Clin Microbiol. 2012;50:312-7.

29. Rooney PJ, O'Leary MC, Loughrey AC, McCalmont M, Smyth B, Donaghy P. Nursing homes as a reservoir of extended-spectrum beta-lactamse (ESBL)-producing ciprofloxacin-resistant Escherichia coli. J Antimicrob Chemother.2009;64: 635-41.

30. Freeman JT, Gormack S, De Almeida MN, Roberts SA. Natural history of rectal colonization with extended-spectrum betalactamase producing Enterobacteriaceae: a retrospective review with up to 6 years of follow-up. Healthcare Infection. 2013;18:1525. https://doi.org/10.1071/HI13013.

31. Thouverez M, Talon D, Bertrand X. Control of Enterobacteriaceae producing extended-spectrum beta-lactamase in intensive care units: rectal screening may not be needed in non-epidemic situations. Infect Control Hosp Epidemiol. 2004;25:838-41.

32. Zahar JR, Lortholary O, Martin C, Potel G, Plesiat P, Nordmann P. Addresssing the challenge of extended-spectrum beta-lactamases. Curr Opin Invest Drugs. 2009;10:172-80.

33. Demiraslan H, Cevahir F, Berk E, Metan G, Cetin M, Alp E. Is surveillance for colonization of carbapenem-resistant gram-negative bacteria important in adult bone marrow transplantation units? Am J Infect Control. 2017;45:735-9.

34. Zimmerman FS., Assous MV, Bdolah-Abram T, Lachish T, Yinnon AM, Wiener-Well Y. Duration of carriage of carbapenem-resistant Enterobacteriaceae following hospital discharge. Am J Infect Control. 2013;41:190-4.

35. Zalecenia dotyczące postępowania w przypadku identyfikacji w zakładach opieki zdrowotnej szczepów bakterii Enterobacteriaceae wytwarzających karbapenemazy typu KPC. http://www.antybiotyki. edu.pl/pdf/KPC-20100305. 